\title{
Digital Models for Arterial Pressure and Respiratory Waveforms
}

\author{
Ivaturi S. N. Murthy and Gullapalli Sita
}

\begin{abstract}
Digital models for arterial pressure pulse (APP) and respiratory volume waveforms $(\mathrm{RVW})$ are proposed for efficien representation of these signals. When these signals are discrete cosine transformed (DCT), the pole-zero technique of SteiglitzMcBride (SM) gave system functions of much lower order than those obtained directly from the signals. The DCT of a bell-shaped biphasic wave needed two poles and two zeros. Based on this, the model order is fixed by the number of distinct peaks in the magnitude spectrum of the transformed APP/RVW signal. The partial fraction expansion (PFE) of the system function allowed delineation of component waves present in the time signal. The angles of model poles and zeros enabled easy determination of several important features from both of these signals. The model performance is evaluated using the normalized root mean-square error (NRMSE). A Bayes classifier using the pole angles as the feature vector performed satisfactorily when a limited number of RVW's recorded under deep and rapid maneuver are classified into normal $(n)$ and abnormal $(u b)$ categories of respiratory pathways.
\end{abstract}

\section{INTRODUCTION}

A RTERIAL pressure pulse (APP) and respiratory volume versus time waveforms (RVW) are among several other signals generated in the physiological system with characteristic features in the form of peaks and valleys, and have been extensively used in clinical studies [1], [2], [11]. One goal behind the analysis of all such signals with digital pattern recognition and system identification methods has been to infer the functional status of the underlying physiological system [2]. Changes in the pulse wave contour were found to be useful for the identification of hypertension, peripheral arteriosclerosis, diabetes [3], and for monitoring patients in the intensive care units (ICU's) [6]. These applications however, demand identification of important points such as beginning, peak, and end systole, beginning of dicrotic notch, and end diastole [4], [5]. Pryor [8] used a single weighted sum, while DeBakey and Glaeser [9] adopted the AZTEC processor for the identification of some of these features. In patient monitoring applications, these features enable us to determine the pulse interval or rate, the foot-to-systolic, foot-to-dictoric notch, and ejection time periods for every heart cycle [4], [5]. Warner [7] divided the APP into four time intervals for estimating stroke volume (SV) and cardiac output (CO), two important indexes of the left ventricular function.

While the pattern of the APP wave gives an indication of the performance of the arterial circulatory system, the

Manuscript received July 1, 1991; revised August 7, 1992.

The authors are with the Department of Electrical Engineering, Indian Institute of Science, Bangalore 560 012, India.

IEEE Log Number 9209293. respiratory volume versus time waveform (RVW) is useful for the evaluation of respiratory mechanics and for detecting abnormalities of respiratory pathways. Analysis of breathing patterns in acutely ill patients reveals distinct differences from those observed in normal subjects [11]. One has to determine significant points such as end of inspiration and end of expiration to quantify these waveforms in terms of tidal volume $\left(V_{T}\right)$, frequency $(f)$, minute ventilation $\left(V_{e}\right)$, and the ratio $\left(T_{I} / T_{\text {tot }}\right)$ (inspiratory time/total cycle time of ventilation) called inspiratory duty cycle. Splitting a breathing cycle into its components may yield information unobtainable through analysis of the feature set $\left(V_{T}, f, V_{e}\right)$. Identification methods based on analog and Compartmental models of lung mechanics are found to be helpful for the detection and diagnosis of pulmonary diseases, monitoring patient status, and to study the effects of therapy and control [10]. In [12], it is shown that frequency and time domain analysis of air flow breath patterns helps to detect patients with chronic obstructive airway disease. Rao et al. [13] carried out spectral analysis of respired volume waveforms obtained under tidal (NB), deep and rapid (DR), and rapid and shallow (RS) maneuvers for detecting airway obstruction (AWO) and airway restriction (AWR) abnormalities of respiratory pathways.

In the present paper, our main objective is the extraction of clinically significant features from a given APP signal, while signal classification is the primary goal in the case of RVW using a common framework of the system theoretic point of view. To this end, we show that the DCT of a bellshaped biphasic wave can be pole-zero modeled with a system function of order $(2,2)$ using the Steiglitz-McBride (SM) method. Conversely, the inverse discrete cosine transform (IDCT) of the model impulse response gives back the time signal with all its features intact. Motivated by this result, we develop pole-zero models to both APP and RVW signals which contain such bell-shaped waveforms, and then proceed to use the model parameters in the $\mathrm{z}$-plane to meet the above two objectives. We also demonstrate the advantages of modeling the DCT transformed signal over modeling the direct time signal. The performance of the model is quantified in terms of the normalized root mean-square error (NRMSE) in percent between the signal and model output and the deviation of extracted features from the actual values. A scheme is proposed for the classification of RVW into normal $(n)$ and abnormal $(a b)$ categories using the pole angles as the feature vector.

In Section II, the transform representation of a biphasic wave, the rationale behind the theory of modeling APP and 
RVW, and the delineation of a signal into its components are explained. The performance criteria for evaluation of the model and databases used are considered in Section III, while the results of analysis of APP and RVW are dealt with in Section IV. Conclusions and discussion are included in the final section.

\section{THEORY}

\section{A. Definition of DCT and IDCT}

Of the four forms of the discrete cosine transform (DCT) of a discrete-time sequence $x(n), n=0,1,2, \ldots, N-1$,i.e., of $N$ sample duration, we use the following definition [14]:

$$
X(k)=\sqrt{(2 / N)} C_{k} \sum_{n=0}^{N-1} x(n) \cos \left[\frac{(2 n+1) k \pi}{2 N}\right]
$$

where

$$
\begin{aligned}
C_{k} & =1 / \sqrt{2} & & k=0 \\
& =1 & & \text { otherwise. }
\end{aligned}
$$

The inverse transform (IDCT) of $X(k)$ in (1) is

$$
x(n)=\sqrt{(2 / N)} \sum_{k=0}^{N-1} C_{k} X(k) \cos \left[\frac{(2 n+1) k \pi}{2 N}\right] .
$$

We also make use of the linearity property of the DCT.

\section{B. Representation and Discrete Cosine Transformation of a Biphasic Wave}

Consider a second-order discrete-time system with a complex pole pair at $r \nless \theta$ and two real zeros $a_{1}$ and $a_{2}$ in the $z$ plane. The transfer function of such a system having a gain $\mathrm{G}$ is given by

$$
X(z)=\frac{\mathrm{G}\left(1-a_{1} z^{-1}\right)\left(1-a_{2} z^{-1}\right)}{1-2 r \cos \theta z^{-1}+r^{2} z^{-2}}
$$

which, in turn, can be rewritten as

$$
X(z)=c+\frac{a+b z^{-1}}{1-2 r(\cos \theta) z^{-1}+r^{2} z^{-2}}
$$

where $\mathrm{c}=a_{1} a_{2} G / r^{2} ; a=G-c, b=2 c r \cos \theta-\left(a_{1}+a_{2}\right) G$. It can be shown that $X(k)$, the impulse response of the transfer function $X(z)$ in (4), is a damped cosinusoid and is given by

$$
\begin{aligned}
X(k)= & c \delta(k)+\left(a r^{k} / \sin \theta\right) \sin ((k+1) \theta) \\
& +\left(b r^{k-1} / \sin \theta\right) \sin k \theta
\end{aligned}
$$

The IDCT of sequence $X(k)$ after some trigonometric simplification is given by

$$
\begin{aligned}
x(n)= & \frac{c+a(1-\sqrt{2})}{\sqrt{N}}+\frac{1}{\sqrt{(2 N)} \sin \theta} \\
& \cdot\left[\frac{\mathrm{a} \sin \theta+a r \sin \mathrm{a}+b \sin (a+\theta)}{1+r^{2}-2 r \cos (a+\theta)}\right. \\
& \left.+\frac{a \sin \theta-a r \sin \mathrm{a}-b \sin (a-\theta)}{1+r^{2}-2 r \cos (a-\theta)}\right]
\end{aligned}
$$

where

$$
a=\frac{(2 n+1) \pi}{2 N} .
$$

In the derivation of (6), terms with $r^{N-1}, r^{N}, r^{N+1}$ are neglected since $r<1$. The parameter set $P(r, \theta a, b, \mathrm{c})$ in (4) controls the frequency of oscillation of $X(k)$ in $(5)$ and the shape of the biphasic wave $x(n)$ in (6) as well as $x_{p}$, the location of the sample with the largest amplitude called the peak sample number. To illustrate this point, consider the parameter sets

$$
\begin{aligned}
& P_{\mathrm{I}}=\left[\begin{array}{lllll}
0.6374 & 0.5972 & 8.8931 & -6.7584, & -0.27701
\end{array}\right. \\
& P_{\mathrm{II}}=\left[\begin{array}{lllll}
0.9269 & 1.3951 & 0.0711 & -0.3315, & -0.27701(7 \mathrm{a})
\end{array}\right. \\
& \text { and } \\
& P_{\text {III }}=\left[\begin{array}{lllll}
0.7094 & 1.1382 & 3.4234 & -3.0781 & 0.05671
\end{array}\right. \\
& P_{\mathrm{IV}}=\left[\begin{array}{llllll}
0.7517 & 2.3530 & 0.0349 & 1.3099 & 0.05671 & \text { (7b) }
\end{array}\right.
\end{aligned}
$$

in (4). The resulting impulse responses $X_{i}(k), i=\mathrm{I}$, II, III, IV computed from (5) are shown in Fig. 1(a), (b) and 1(e), (f), respectively. The corresponding responses $x_{i}(n)$ computed from (6) are shown by curves I, II in Fig. 1(c) and III, IV in Fig. 1(g), respectively. This shows that a bell-shaped biphasic wave can be generated by taking the IDCT of the impulse response of a system of order $(2,2)$ and vice versa. The sum of curves I, II and III, IV shown in Fig. 1(d) and (h), respectively, results in APP and RVW signals.

\section{Modeling}

For a discrete-time signal, assume a parametric model in the form of a linear difference equation,

$$
x(n)+\sum_{i=1}^{p} a_{i} h(n-i)=\sum_{j=0}^{q} b_{j} \delta(n-j)
$$

or in the $z$ domain,

$$
X(z)=\frac{B(z)}{A(z)}=\frac{b_{0}+b_{1} z^{-1}+\cdots+b_{q} z^{-q}}{1+a_{1} z^{-1}+\cdots+a_{p} z^{-p}} .
$$

When the input $\delta(n)$ is an impulse, the model output $x(n), n=0,1,2, \ldots, N-1$, represents the $N$ significant samples in the impulse response (of infinite length) of the unknown system of order $(p, q)$ and is an approximation to $x(n)$. Although there are several methods, such as the Akaike information criterion, available in the literature [15], there is no unique way to determine the model order, which is usually signal dependent. We used a simple heuristic procedure to determine the same (see Section II-F below). From among several efficient algorithms, we have selected the methods due to Shanks [16] and Steiglitz-McBride (SM) [17] in the present study for estimating $B(z)$ and $A(z)$, the numerator and denominator polynomials in (9). The system poles and zeros are obtained by solving for the roots of $A(z)$ and $B(z)$. For the purpose of comparison, both the APP signal and its DCT are modeled using the Shanks and the SM methods as detailed below. 1) Model APP signal $x(n)$ by the SM method to obtain $\hat{X}(z)$. Compute the impulse response of the model to obtain $\hat{x}(n)$. In this case, the DCT and IDCT blocks in Fig. 2 have to be ignored. 2) Model $X(k)$, the DCT of APP signal $x(n)$ to get $\hat{X}_{1}(z)$ and obtain $\hat{x}_{1}(n)$, the reconstructed output, 


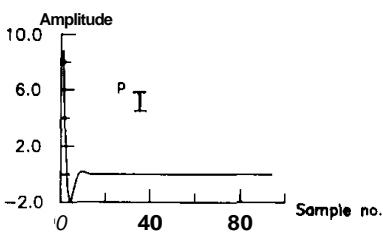

(a)

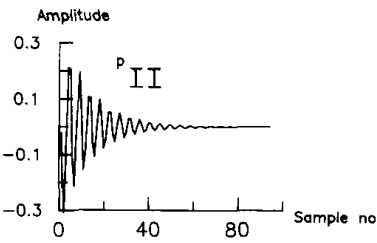

(b)

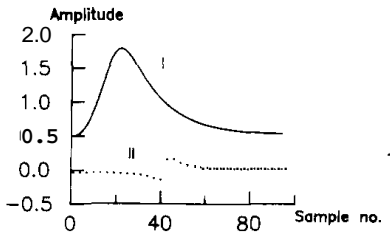

(c)

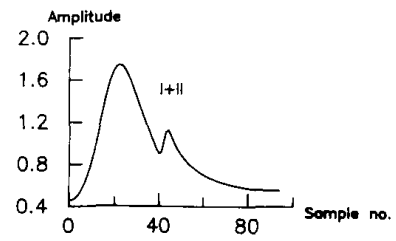

(d)

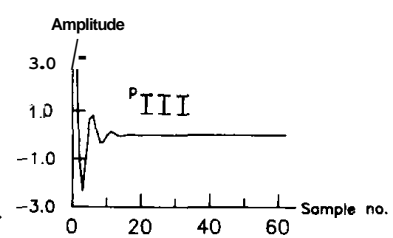

(e)

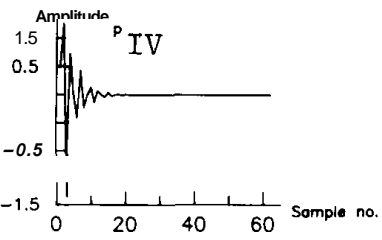

(f)

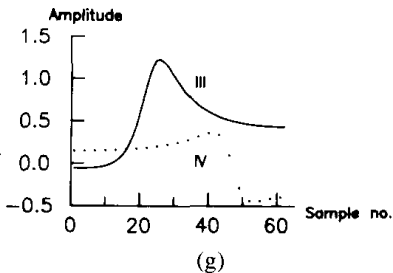

(g)

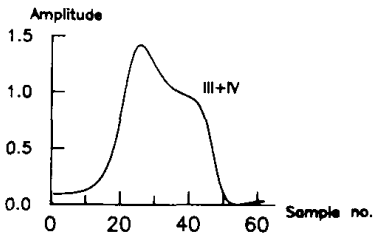

(h)
Fig. 1. (a) Impulse response of a system of order $(2,2)$ with parameter set $p_{\mathrm{I}}(0.6374,0.5972,8.8931,-6.7584,-0.2770)$. (b) Impulse response of a system of order $(2,2)$ with parameter set $p_{\text {II }}(0.9269,1.3951,0.0711$, $-0.3315,-0.2770$ ). (c) (I) IDCT of impulse response of (a) in solid line. (11) IDCT of impulse response of (b) in dotted line. (d) Sum of components (I) and (II) in (c) resulting in an APP signal. (e) Impulse response of a system of order $(2,2)$ with parameter set $p_{\mathrm{III}}(0.7094,1.1382,3.4234,-3.0781,0.0567)$. (f) Impulse response of a system of order $(2,2)$ with parameter set $p_{I V}(0.7517$ $2.3530,0.0349,1.3099,0.0567)$. (g) (III) IDCT of impulse response of (e). IV) IDCT of impulse response of (f). (h) Sum of components (III) and (IV) in $(\mathrm{g})$ resulting in an RVW signal.

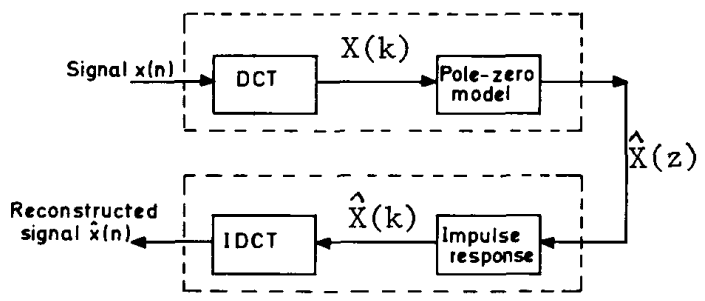

Fig. 2. Block diagram for modeling procedure.

by computing the IDCT of $\hat{X}_{1}(k)$, the impulse response of the model. This is shown in block diagram of Fig. 2. Repeat Steps 1) and 2) using the Shanks method. The whole procedure is then repeated for the RVW. The SM method estimates model parameters iteratively, whereas the Shanks method does so noniteratively in one shot.

\section{Delineation of Overlapping Biphasic Waves in $x(n)$}

In Section II-B, we saw that a bell-shaped biphasic wave can be modeled by a function of order $(2,2)$. Let us now add two such biphasic waves I, II and III, IV that overlap with each other, as shown in Fig. 1(c) and (g), to get the resultants shown in Fig. 1(d), (h), respectively. By extending the previous argument in Section II-C, $X(k)$, the DCT of $x(n)$, can be modeled by a system function $\hat{X}(z)$ of order $(4,4)$. As the peaks of the two overlapping biphasic waves come close to each other, the two pole angles in the model also move close to each other and vice versa. Further, we can expand $\hat{X}(z)$ into two fractions $\hat{X}_{\mathrm{I}}(z)$ and $\hat{X}_{\mathrm{II}}(z)$, each of order $(2,2)$, by the partial fraction expansion technique (PFE). The two component waves $\hat{x}_{i}(n), i=\mathrm{I}$, II of which signal $x(n)$ is composed, are then obtained by computing the IDCT of the impulse response of each of these fractions. The same theory holds for curves 111 and IV in Fig. 1(g), and their sum gives the RVW in Fig. 1(h). This theory can be extended to split a signal $x(n)$ that contains $M$ biphasic waves into its respective components, whether overlapping with each other or not, via the transform domain.

\section{E. Relationship Between $\theta$ the Pole Angle and $x_{p}$ the Peak Sample Number}

If $x_{i}(n)=x(n) \delta(n-i)$, an impulse of amplitude $x_{i}$ occurring at time $\mathrm{n}=i, \mathrm{i}=0,1,2, \cdots, \mathrm{N}-1$, then from (1), $X_{i}(k)$ is an undamped cosine wave with amplitude $A_{i}=$ $\left[\sqrt{(2 / N)} x_{i}\right]$ and frequency of oscillation $w_{i}=[(2 i+1) \pi / 2 N]$ rad. As can be seen, both $\boldsymbol{A}$; and $w_{i}$ are functions of the sample number $i$ where the pulse is located in the time domain. The cosine wave with an undamped envelope (in dotted line) in Fig. 3(b) is the DCT of the impulse at $i=4$ in Fig. 3(a). When we model this undamped cosine wave, it is represented by a complex pole on the unit circle in the $z$ plane, i.e., by a secondorder system function. The pole angle $\theta$ is determined from the frequency of oscillation $w_{i}$. But $\mathrm{w}$; in turn, is a function of the sample number $i$. Thus when the impulse is shifted from $i=0$ to $\mathrm{N}-1$ in the time domain, the frequency of oscillation of $X(k)$ changes from $(\pi / 2 N)$ to $[(2 N-1) \pi / 2 N] \mathrm{rad}$ and the pole angle $\theta$ changes from 0 to $180^{\circ}$ in the $z$ domain.

We note from (1) that any two adjacent samples in the time domain give rise to cosinusoids whose frequencies of oscillation differ by $(\pi / N)$ rad in the DCT domain. If a signal $x(n)$ of length $N$ samples, $n=0,1, \ldots, N-1$, contains a bell-shaped biphasic wave with $n_{2}-n_{1}+1$ consecutive nonzero samples ( $n_{1}$ and $n_{2}$ are arbitrary) with amplitudes $x_{i}, i=n_{1}, n_{1}+1, \cdots, n_{2}-1, n_{2}$, and $x_{i}=0$ for $i \neq$ $n_{1}, n_{1}+1 . \ldots, n_{2}$, then the DCT is the sum of that many undamped cosinusoids with amplitudes $\left[\sqrt{(2 / N)} x_{i}\right]$. This sum results in a damped cosinusoid in $X(k)$ with its frequency of oscillation very nearly the same as that due to the peak sample number $x_{p}$ in $x(n)$, viz. $\left[\left(2 x_{p}+1\right) \pi / 2 N\right]$. For the biphasic wave $x_{i}(n)$ with $i=100, \ldots, 150$ in Fig. 3(c), $X(k)$, the DCT which is a damped cosine wave (with envelope in dotted line) shown in Fig. 3(d) can be modeled with a system function of order $(2,2)$. In this case, the complex pole at $r \angle \theta$ lies inside the unit circle with an approximate frequency 
$\left(\pi x_{p} / N\right)$ rad in the $z$ plane. Note that this quantity is a close approximation to the frequency of oscillation $[(22,+1) \pi / 2 N]$ of the largest cosinusoid in the DCT of the bisphasic wave under consideration. As we shift the peak sample number $x_{p}$ from $p=0$ to $N-1, \theta$ changes from 0 to $180^{\circ}$ in the $z$ domain.

Let there be $M$ biphasic component waves in a signal $x(n)$ of length $N$ samples with their peak sample numbers at $m_{k}, k=1,2, \ldots, M$. Angle $\theta_{k}$ subtended by the pole of the kth component at the origin with the positive real axis in the $z$ plane is given by $\theta_{k}=\left[\left(2 x_{k}+1\right) \pi / 2 N\right]$ rad. Therefore, the peak sample number of the kth biphasic wave can be approximated by the relation

$$
m_{k} \approx\left(\theta_{k} / \Pi\right) N
$$

The number of samples $N$ being a constant, $m_{k}$ and $\theta_{k}$ are directly related.

\section{F. Comparison of Spectra and Model Order Determination}

One can compute the spectra $X(w), X(\Omega)$ of the signals $x(n)$ and $X(k)$ as well as $\hat{X}(w), \hat{X}(\Omega)$, the spectra of $\hat{x}(n) \boldsymbol{X}(\mathrm{k})$, the reconstructed signals from the models, respectively. We note that $X(w)$ in Figs. 4(b), 6(b) do not show spectral features such as peaks and valleys, and therefore do not provide any indication of the order $(p, q)$ to be used to model $x(n)$. We therefore used models of increasing orders starting from $(\mathbf{2}, \mathbf{2})$ and going to $(10,10)$ in steps of $(\mathbf{2}, \mathbf{2})$ to determine that order which minimizes the NRMSE. On the other hand, $X(\Omega)$ in Figs. 5(c), 7(c) show two clear peaks $(n=2)$ indicative of the number of biphasic component waves present in $x(n)$, and allows easy determination of order $(2 n, 2 n)$, required to model signal $X(k)$. In actual applications, it was found that the order required to model $X(k)$ is much lower than that required by $x(n)$. Further, the match between $X(\Omega)$ and $\hat{X}(\Omega)$ is far better than the one between $X(w)$ and $\hat{X}(w)$. A good spectral match is a very desirable feature in all modeling applications. In addition, when we model $X(k)$, there is a one-to-one correspondence between a model pole and a biphasic component in $\hat{x}(n)$ or $x(n)$, whereas the model of $x(n)$ does not reveal any such close relationship. This aspect is particularly significant as it allows delineation and study of the individual components that are present in $x(n)$. These are distinct advantages when we model $X(k)$, the DCT transformed signal over modeling the direct time signal $x(n)$.

\section{G. Bayes Classifier for Respiratory Waveforms}

For the purposes of uniformity, signals of different amplitudes and lengths have been normalized before modeling. The length of all signals considered for analysis is taken as that of the largest length in the database, say $N$, and that of the largest amplitude as 1 . If a signal has fewer samples in it, an appropriate number of zeros is added at its tail end to make its length equal to $N$. These two steps allow comparison of various types of signals without altering the shape or features present in them. All the normalized signals (both normal and abnormal with respiratory path diseases) are modeled with a function of order $(2 n, 2 n)$, and a heuristic feature vector $\mathrm{X}=\left[\theta_{1}, \theta_{2}, \ldots, \ldots, \theta_{n}\right]$ is formed using the complex conjugate pole angles $\theta_{1} \theta_{2}, \ldots, \theta_{n}$ obtained from the model. A part of the database is used as the training set and the remaining as the test set. Assuming that the probability density functions (pdf's) are Gaussian with means $m_{n}$ and $m_{a b}$ and covariances $k_{n}$ and $k_{a b}$ for the $n$ 's and $a b$ 's, respectively, the test set in the database is classified using a Bayes classifier [18] with the following discriminant function $g(X)$ :

$$
\begin{aligned}
g(x)= & \left(x-m_{a b}\right)^{T} K_{a b}^{-1}\left(x-m_{a b}\right) \\
& -\left(x-m_{n}\right)^{T} K_{n}^{-1}\left(x-m_{n}\right) \\
& +\ln \frac{\left|K_{a b}\right|}{\left|K_{n}\right|}-2 \ln \frac{P C_{a b}\left(C_{a b}\right)}{P C_{n}\left(C_{n}\right)}
\end{aligned}
$$

where $C_{a b}$ and $C_{n}$ denote abnormal and normal classes, respectively.

The means and variances are estimated from

$$
\bar{m}_{j}=1 / N_{(j)} \sum_{i=1}^{N_{(j)}} \bar{x}_{i}
$$

and

$$
\begin{gathered}
K_{(j)}=1 / N_{(j)} \sum_{i=1}^{N_{(j)}}\left(\bar{x}_{i}-\bar{m}_{(j)}\right)\left(\bar{x}_{i}-\bar{m}_{(j)}\right)^{T} \\
j=\text { nor } a b \text { class }
\end{gathered}
$$

$N_{(j)}$ is the number of elements in any one class.

Due to the limited number of signals in RVW database, we used the leave-one-out or $U$ method [19] to estimate the correct classification by the Bayes classifier.

\section{DATA ACQUiSITION AND EVALUATION}

\section{A. Data Acquisition}

The data have been acquired from two sources. APP signals from 20 patients undergoing surgical procedures at the National Institute of Mental Health and Neurosciences (NIMHANS), Bangalore, India, were recorded on an FM tape recorder (Schlumberger MP 5501) for periods ranging from 15 to $30 \mathrm{~min}$. These signals are digitized at 100 samples/s (sps) with $12 \mathrm{~b}$ accuracy on a PDP-11 hybrid computer in standalone mode. About 50\% of the RVW's were obtained from the respiratory database built by the ECE Department of Osmania University, Hyderabad, India, and the rest from patients at NIMHANS, all under deep and rapid (DR) maneuver.

\section{B. Performance Evaluation}

The criteria used for the evaluation of the algorithms are: 1) the NRMSE in percent, and 2) the error between the location of the peak sample number determined visually in a time domain component wave and the one estimated from the model using (10). The normalized root mean-square error (NRMSE) in 

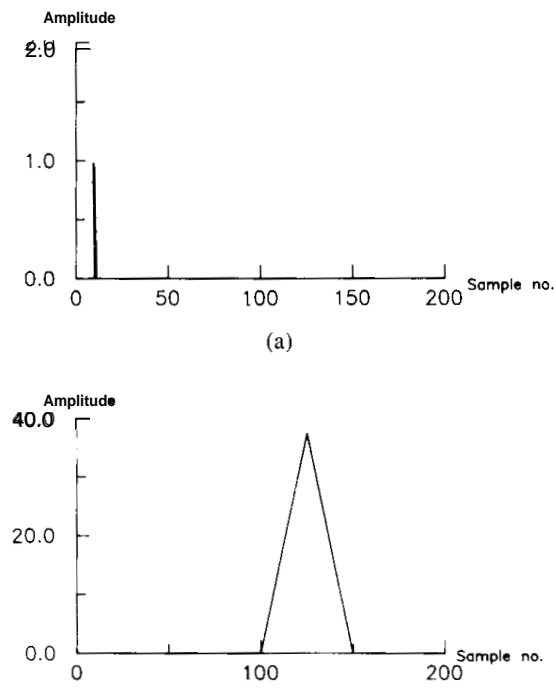

(c)

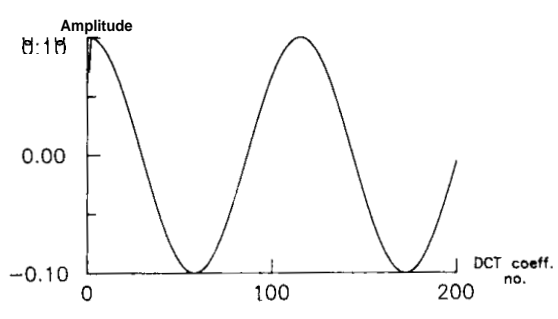

(b)

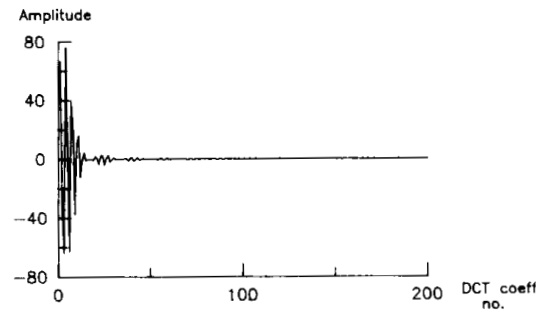

(d)

Fig. 3. (a) An impulse at sample number $n=4$. (b) DCT and undamped envelope of the impulse shown in (a). (c) Biphasic wave with unequal slopes between sample numbers 100-150. (d) DCT of biphasic wave shown in (c).

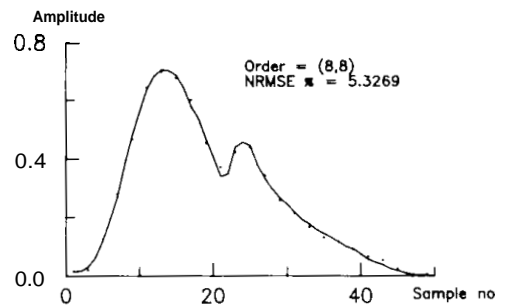

(a)

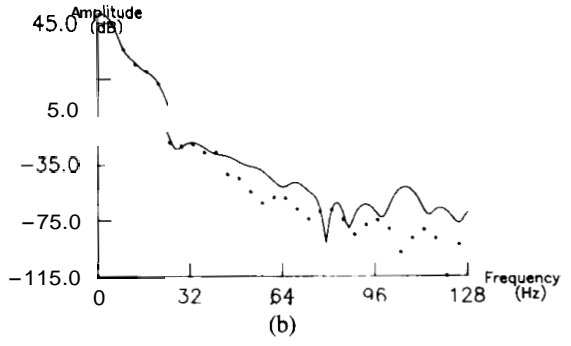

ZERO $O$

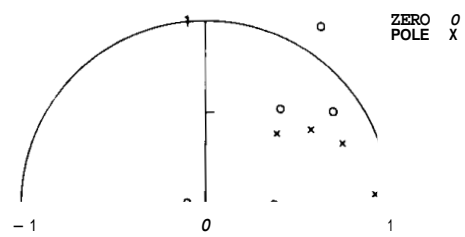

(c)

Fig. 4. (a) APP signal (solid line) and the model output (dotted line) derived directly from time signal. (b) Spectra of the signals in (a). : original, . . . . . . Model output. (c) Pole-zero plot in $z$ plane for the signal in (a).

percent is defined as

$$
\% \text { NRMSE }=\left[\frac{\sum_{i=0}^{N-1}[x(i)-\hat{x}(i)]^{2}}{\sum_{i=0}^{N-1}[x(i)]^{2}}\right] \times 100
$$

where $x(i)$ and $\hat{x}(i)$ are the actual and reconstructed signals and $N$ is their length. In addition, we used two more comparisons: the match between spectra of: 3 ) the time signal $x(n)$ and that of the output $\hat{x}(n)$ obtained from the model derived directly from the signal $x(n)$ itself, and 4) the transformed signal $X(k)$ and that of the model output [obtained either from (4) or (5)].

\section{RESULTS}

\section{A. Arterial Pressure Pulse (APP)}

The arterial pressure pulse (APP) shown in Fig. 4(a) has two peaks, first, the systolic peak with a larger amplitude, and second, the dicrotic notch with a relatively small value. When one attempts to model this signal, the order to be used 

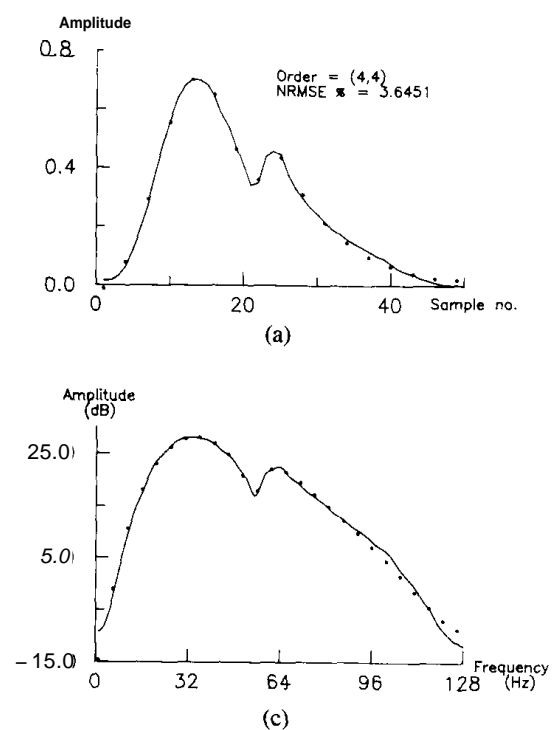
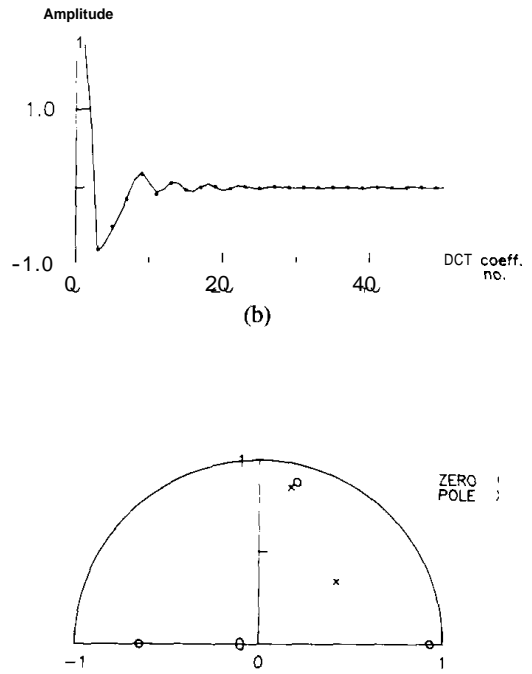

(d)

Fig. 5. APP signal (solid line) and the model output (dotted line) derived from the DCT transformed signal. (b) DCT of signal (solid line) in (a) obtained with (1) and impulse response of model (dotted line) estimated with (5). (c) Spectra of signals in (b) for the transformed signal (solid line) and model output (dotted line). (d) Pole-zero plot obtained from the transformed signal in (b).

is not at all apparent either from $x(n)$ or its spectrum $X(w)$ in Fig. 4(b). Therefore, as discussed in Section II-F, this time waveform of 48 samples in length is modeled using both the Shanks and SM methods with model orders starting from $(2$, 2) up to $(10,10)$ in steps of $(2,2)$. The NRMSE in percent between the signal $x(n)$ and the model output $\hat{x}(n)$ computed using (14) attained its minimum (5.3269\%) for the model order of $(8,8)$ for the SM method. The model output (in dotted line) is superimposed on the original signal in Fig. 4(a). The spectra of these two signals are shown in Fig. 4(b) in continuous and dotted lines, respectively. The results obtained by the Shanks method compared poorer than those derived by the SM method and are not shown here. For some of the signals, a much higher order of about $(15,15)$ is required to get a good fit between the signal and the model output. Note the high model order required, as well as the apparent mismatch between the two spectra after a few initial samples, as seen from Fig. 4(b). The pole-zero plot in the $z$ plane for the example considered is shown in Fig. 4(c) with two complex zeros and one real zero lying outside the unit circle. There is no apparent relationship between the waveform features and poles (zeros) of the model.

As discussed in Section II-D, the APP may be considered as a juxtaposition of two overlapping biphasic waves. $X(k)$, the DCT of $x(n)$, is modeled using a function of order $(4,4)$, i.e., $n=\mathbf{2}$, as confirmed by the number of peaks in $X(\Omega)$ in Fig. $5(c)$. Thus, the order fixation is simplified to the identification of the number of biphasic waves in $x(n)$ or the number of peaks in $|X(\Omega)|$, the magnitude spectrum of $X(k)$. The time signal (continuous line) and the model output (dotted line) are shown in Fig. 5(a) for comparison. Both the prominent systolic peak and dicrotic notch got modeled. Fig. 5(b) shows $X(k)$, the DCT of the time signal (continuous line), and $X(k)$, the impulse response (dotted line) of the model using the $\mathbf{S M}$ method, while the spectra of the signals are shown in Fig. 5(c) in continuous and dotted lines, respectively. Note the excellent match in this case among the signals, the DCT's, and their spectra. Further, the NRMSE in percent in this case is 3.6451 for model order $(4,4)$. The system poles and zeros in the $z$ plane are shown in Fig. 5(d). Note that there are two complex pole pairs, one corresponding to the systolic peak at $\theta_{1}=38.6^{\circ}$, and the other due to the dicrotic notch at $\theta_{2}=78.57^{\circ}$, one complex zero corresponding to the beginning of dicrotic notch at 76.77' and two real zeros, one on the right and another on the left of the origin in the $z$ plane. When the transformed signal in Fig. 5(b) is modeled with a system of order $(2,2)$, only the systolic peak got modeled with pole angle $\theta_{1}^{\prime}$ as this is the predominant one over the dicrotic notch. This pole angle coincides with $\theta_{1}$, the one corresponding to the systolic peak as obtained with order $(4,4)$.

The sample numbers corresponding to peak systole and dicrotic notch as determined from pole angles $\theta_{1}$ and $\theta_{2}$, respectively, using (10) differed from the actual ones [determined visually from the time signal $x(n)]$ by at most two samples. The onset of dicrotic notch is determined from the complex zero. If the signal is somewhat flat instead of having a clear and sharp peak, the pressure pulse may require a higher order function for a good reproduction of the signal. The mean results from the 20 APP signals are presented in Table I.

\section{B. Respiratory Waveforms}

The modeling procedure is now extended to these signals with an aim to determine the beginning and end of inspiration as well as end of expiration. Consider a typical respiratory waveform recorded under deep and rapid (DR) maneuver from a normal subject, as shown in Fig. 6(a) (continuous line). This signal is modeled by both the Shanks and Steiglitz-McBride methods from orders $(2,2)$ up to $(10,10)$ in steps of $(2,2)$. 
TABLE I

\begin{tabular}{|c|c|c|c|c|c|c|c|c|c|c|}
\hline \multicolumn{7}{|c|}{ Arterial Pressure Pulse (APP) } & \multicolumn{4}{|c|}{ Respiratory Volume Waveform (RVW) } \\
\hline & Systolic & Sample No. & $\begin{array}{l}\text { Beginn } \\
\text { Dicroti }\end{array}$ & nple No. of & $\begin{array}{l}\text { Peak S } \\
\text { Dicroti }\end{array}$ & $\begin{array}{l}\text { No. of } \\
\mathrm{ch}\end{array}$ & First $\mathrm{P}$ & nple No. & Second & ample No. \\
\hline Mean & $\begin{array}{l}\text { Actual } \\
21.42\end{array}$ & $\begin{array}{l}\text { Calculated } \\
20.82\end{array}$ & $\begin{array}{l}\text { Actual } \\
\mathbf{3 0 . 8 5}\end{array}$ & $\begin{array}{l}\text { Calculated } \\
31.19\end{array}$ & $\begin{array}{l}\text { Actual } \\
\text { 33.42 }\end{array}$ & $\begin{array}{l}\text { Calculated } \\
32.06\end{array}$ & $\begin{array}{l}\text { Actual } \\
16.9\end{array}$ & $\begin{array}{l}\text { Calculated } \\
16.6\end{array}$ & $\begin{array}{l}\text { Actual } \\
33.5\end{array}$ & $\begin{array}{l}\text { Calculated } \\
33.79\end{array}$ \\
\hline Variance & 9.67 & 11.33 & 6.12 & 8.42 & 7.38 & 8.29 & 17.69 & 22.0 & 52.45 & 48.88 \\
\hline
\end{tabular}

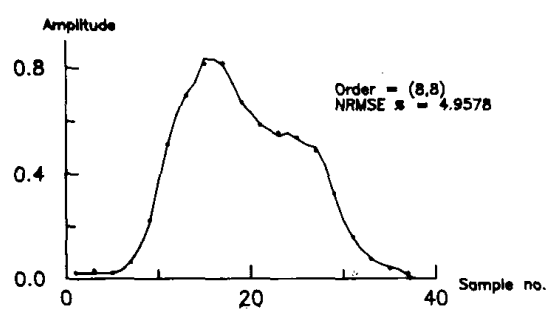

(a)

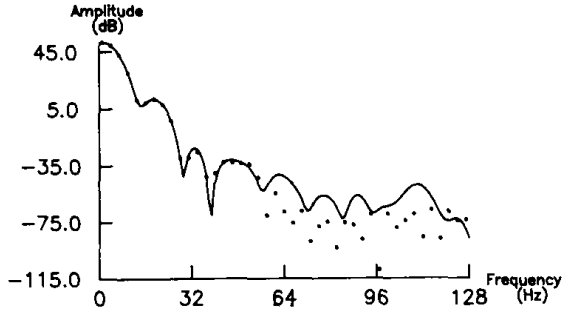

(b)

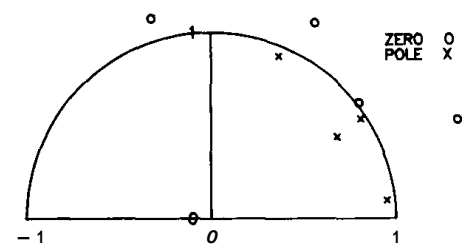

(c)

Fig. 6. (a) RVW signal (solid line) and the model output (dotted line) derived directly from the time signal. (b) Spectra of the signals in (a). : original signal, ....... model output. (c) Pole-zero plot in $z$ plane for the signal in (a).

The NRMSE in percent for the order $(8,8)$ was $4.957 \%$. Here again, the SM method performed better, and therefore for this model is shown in Fig. 6(a) in dotted line. The curves in Fig. 6 (b) show the corresponding spectra. In this case, also note the spectral mismatch as in Fig. 4(b). The pole-zero plot obtained from the model is shown in Fig. 6(c) with all the four complex zeros lying outside the unit circle.

Fig. 7(a) shows the time signal (continuous line) and the output derived from the model of the transformed signal by the SM method using an order $(4,4)$ (in dotted line). The NRMSE in percent in this case is found to be 2.9. Fig. 7(b) shows $X(k)$, the transformed signal, and $\hat{X}(k)$, the model impulse response, in continuous and dotted lines. Fig. $7(\mathrm{c})$ shows an excellent match between $X(\Omega)$, and $\hat{X}(\Omega)$, the spectra of the transformed signal and that of the model. The pole-zero plot is shown in Fig. 7(d). With the help of pole angles $\theta_{1}$ and $\theta_{2}$, the end of inspiration or beginning of expiration are determined, and are compared with the actual values as determined manually from the time waveform. If one is interested only in the separation of inspiration and expiration phases of the RVW, an order of $(2,2)$ is sufficient. The pole angle gives a sample number which is accurate enough for indicating end of inspiration. An order $(4,4)$, however, gives a better reproduction of the time signal, even when it is not a sharp and peaky one. Again, $\theta_{1}$ gives the sample number corresponding to the end of inspiration. The mean and variance for the sample numbers computed from the model compare well with the corresponding values obtained from the time waveform, and are shown in Table I for both APP and RVW signals.

\section{Component Delineation by Partial Fraction Expansion Technique}

The model in (9), as obtained from the DCT transformed signal by the SM method of both APP and RVW signals in Fig. $1(d)$ and (h) is given by (15) and (16), shown at the bottom of the next page.

When expanded, $\hat{X}_{1}(z)$ and $\hat{X}_{2}(z)$ yield the parameter sets PI, $P_{\text {II }}$ and PIII, $P_{\text {IV }}$ in (7a) and (7b), respectively.

The delineated component waves of the APP signal obtained by taking the IDCT of the impulse responses of the partial fractions are shown in solid (curve I) and dotted lines (curve 11) in Fig. 1(c). It can be seen from these components that the dicrotic notch which begins at sample number 41 coincides with the calculated sample number 41.89 from the zero angle 78.56'. The systolic peak occurs at sample number 20, and the calculated peak systole from the pole angle $\theta_{1}=\mathbf{3 4 . 2 2}$ ' is 18.25. The peak of the dicrotic notch as seen from the time waveform is 43 , and that computed from the pole angle $\theta_{2}=79.93 '$ is 42.62 . Similar analysis is carried out on the entire database, and it was found that the computed sample numbers differed from the actual ones by at most two samples. 


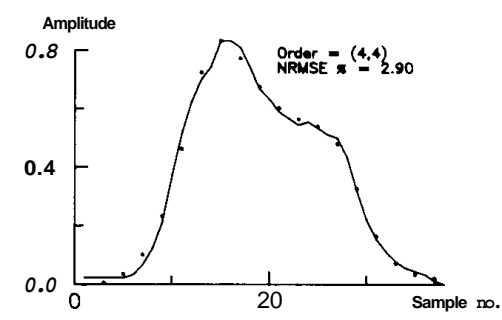

(a)

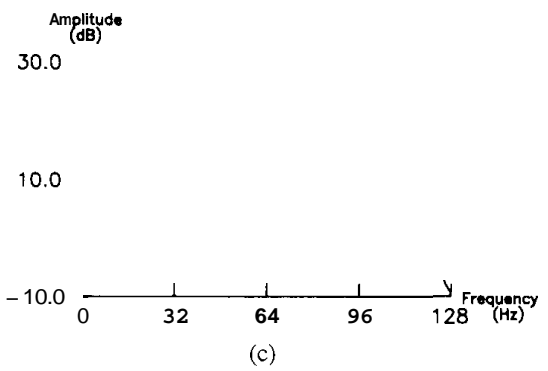

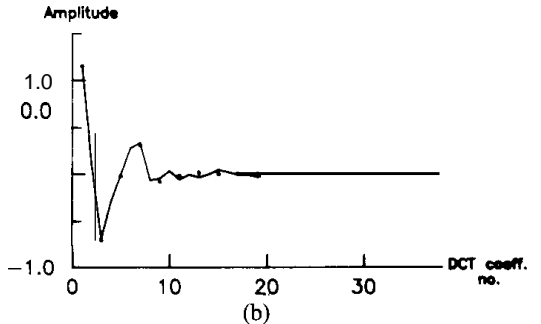

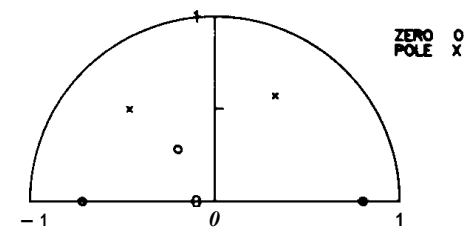

(d)

Fig. 7. (a) RVW signal (solid line) and the model output (dotted line) derived from the DCT transformed signal. (b) DCT of signal (solid line) in (a) and impulse response of model (dotted line). (c) Spectra of signals in (b) for the transformed signal (solid line) and the model output (dotted line). (d) Pole-zero plot in $z$ plane obtained from the transformed signal in (b).

For the respiratory signals, the separation of inspiratory and expiratory phases is carried out using the above analysis, and the results are shown as curves III and IV in Fig. 1(g). The length of the signal considered is 64 samples. The beginning of the inspiratory phase and the start of the respiratory cycle are assumed to be the same. The end of inspiration computed from the pole angle $\theta_{1}=65.21^{\circ}$ is 23.18 samples, which is close to the actual sample number 25 . The variation between the computed sample numbers and the actual values was not more than two for the entire database considered.

\section{Classification of Respiratory Waveforms into n's and ab's}

In the database, we had 26 normal $(n)$ and 13 abnormal $(a b)$ patterns with AWO and AWR as classified by a specialist clinician. The data set is given in Table 11 . We used the leaveone-out method for classifying the data. The discriminant function is modified for each classification of patient with this approach. All the signals, i.e., both $n$ and $a b$ 's, are modeled using an order $(2 n, 2 n)$ with $n=2$ (see Section 11-F), i.e., with order $(4,4)$, after amplitude and length normalization (see Section II-G),

The decision boundary obtained using $g(X)$ is a secondorder curve in the feature space, as shown in Fig. 8. The classification obtained for the data is also shown in the same figure, with normal marked as crosses and abnormal in circles. The classifier detected three false positives marked as 1,2 , and 3 in Fig. 8 and one false negative (marked as 4). To establish the effectiveness of the classifier, however, a large patient population needs to be studied.

\section{CONCLUSION AND DISCUSSION}

\section{A. Conclusion}

The discrete-cosine-transformed APP and RVW signals are pole-zero modeled with lower order system functions than those derived from the time signals directly using the iterative Steiglitz-McBride method. The model order is fixed by counting the number of peaks in $|X(\Omega)|$, the magnitude spectrum of $X(k)$, the transformed signal of $x(n)$, the time signal. The NRMSE computed between the original and reconstructed time signals obtained by inverse transformation (IDCT) of the model impulse response shows that the performance is highly satisfactory. Salient features in the APP, as well as respiratory waveforms extracted from model poles and zeros, are found to match the actual values in the input signals very well. The delineated component waves obtained from a given APPIRVW waveform by partial fraction expansion of the system function enabled detailed study of these signals. Using pole angles of the system function in the z-plane as a feature vector, respiratory waveforms are classified into normal and abnormal categories with good accuracy.

$$
\begin{aligned}
& \hat{X}_{1}(z)=\frac{3.5926+(-2.6435) z^{-1}+(1.5765) z^{-2}+(-0.3689) z^{-3}+(-1.8750) z^{-4}}{1.000+(-1.3782) z^{-1}+(1.6070) z^{-2}+(-1.0373) z^{-3}+(0.3491) z^{-4}} \\
& \hat{X}_{2}(z)=\frac{4.3517+(2.2541) z^{-1}+(-1.6974) z^{-2}+(-0.9040) z^{-3}+(0.2541) z^{-4}}{1.000+(0.4649) z^{-1}+(0.4381) z^{-2}+(0.1972) z^{-3}+(0.2844) z^{-4}}
\end{aligned}
$$


TABLE II

Data Set USED to Design the Classifer

\begin{tabular}{|c|c|c|c|c|}
\hline Patient No. & $\theta_{1}$ & $\theta_{2}$ & Diagnosis & Classification \\
\hline 1 & 16.00 & 37.09 & $n$ & $n$ \\
\hline 2 & 30.83 & 68.72 & $n$ & $n$ \\
\hline 3 & 13.98 & 30.75 & $n$ & $n$ \\
\hline 4 & 26.84 & 55.89 & $n$ & $n$ \\
\hline 5 & 16.27 & 35.24 & $n$ & $n$ \\
\hline 6 & 14.17 & 38.74 & $n$ & $n$ \\
\hline 7 & 21.28 & 58.44 & $n$ & $n$ \\
\hline 8 & 18.64 & 39.12 & $n$ & $n$ \\
\hline 9 & 18.55 & 51.66 & $n$ & $n$ \\
\hline 10 & 35.69 & 64.04 & $n$ & $n$ \\
\hline 11 & 33.19 & 63.92 & $n$ & $n$ \\
\hline 12 & 23.09 & 32.84 & $n$ & $n$ \\
\hline 13 & 32.04 & 35.5 & $n$ & $a b^{*}$ \\
\hline 14 & 26.27 & 33.97 & $a b$ & $a b$ \\
\hline 15 & 24.61 & 48.16 & $a b$ & $a b$ \\
\hline 16 & 26.59 & 36.35 & $a b$ & $a b$ \\
\hline 17 & 27.5 & 49.27 & $a b$ & $a b$ \\
\hline 18 & 28.84 & 51.04 & $a b$ & $a b$ \\
\hline 19 & 31.22 & 49.99 & $a b$ & $a b$ \\
\hline 20 & 35.64 & 50.07 & $a b$ & $a b$ \\
\hline 21 & 36.53 & 52.7 & $a b$ & $a b$ \\
\hline 22 & 11.25 & 61.4 & $n$ & $n$ \\
\hline 23 & 14.72 & 54.22 & $n$ & $n$ \\
\hline 24 & 16.2 & 46.0 & $n$ & $n$ \\
\hline 25 & 28.8 & 41.9 & $a b$ & $a b$ \\
\hline 26 & 14.1 & 48.4 & $n$ & $n$ \\
\hline 27 & 25.28 & 44.57 & $a b$ & $a b$ \\
\hline 28 & 20.21 & 52.23 & $n$ & $n$ \\
\hline 29 & 27.5 & 57.5 & $n$ & $n$ \\
\hline 30 & 26.22 & 27.68 & $n$ & $n$ \\
\hline 31 & 29.46 & 37.83 & $n$ & $a b^{*}$ \\
\hline 32 & 22.73 & 52.01 & $n$ & $n$ \\
\hline 33 & 30.5 & 43.3 & $n$ & $a b^{*}$ \\
\hline 34 & 23.45 & 30.9 & $a b$ & $n^{*}$ \\
\hline 35 & 17.41 & 45.8 & $n$ & $n$ \\
\hline 36 & 27.18 & 47.3 & $a b$ & $a b$ \\
\hline 37 & 24.1 & 42.7 & $a b$ & $a b$ \\
\hline 38 & 30.92 & 67.66 & $n$ & $n$ \\
\hline 39 & 22.56 & 58.1 & $n$ & $n$ \\
\hline \multicolumn{5}{|c|}{$n:$ normal; $a b$ : anormal. } \\
\hline
\end{tabular}

\section{B. Discussion}

Order selection is an important problem, and one can use several criteria, such as the Akaike information criterion [15]. However, none of these methods gave satisfactory results for the signals considered. In the present study, we counted the number of peaks in the magnitude spectrum of $X(k)$, and each peak corresponds to a function of order $(2,2)$. In almost all cases, $X(\Omega)$, the spectra of $X(k)$, the DCT-transformed version of the APP and RVW signals showed two peaks, and as such were modeled with an order $(4,4)$, with an excellent match between the reconstructed and original signals. However, it was found that when the order is overestimated, the extra poles are located on the real axis close to the origin in the $z$-plane, leading to a small bend or kink in $\hat{x}(n)$, the reconstructed signal, and did not cause any dislocation of the peaks, even in the presence of moderate amounts of background noise. When the order chosen is very high, it was noted that the SM algorithm did not converge. When the order is underestimated, the smallest component waves are not modeled and are lost in the reconstructed signal.

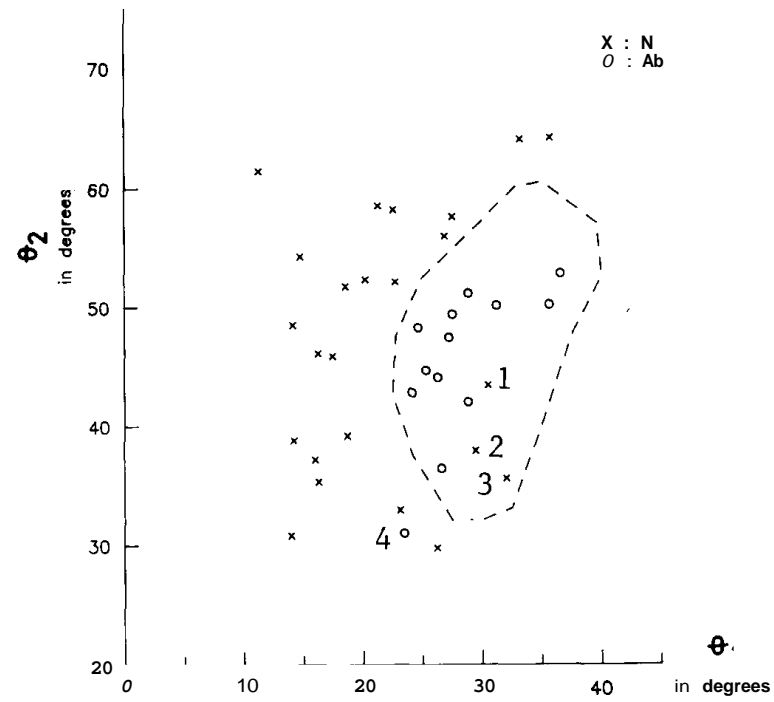

Fig. 8. Decision boundary in the feature space and classification of test set for respiratory waveforms. 1, 2, 3: false positives, 4: false negative.

The SM method needs an initial guess of the poles of the system to commence the iterative process. As is common practice, we also used the linear prediction (LP) coefficients for the initial estimate. When the NRMSE stopped changing significantly, it was assumed that the algorithm had converged. It was found that after about four to six iterations, the NRMSE stopped changing significantly, both for APP and RVW. When it remained less than 0.001 , the algorithm was assumed to have converged. All of the programs have been implemented in Matlab on a PC-386 with a coprocessor.

\section{ACKNOWLEDGMENT}

The authors are grateful to Professor D. C. Reddy, Department of ECE, Osmania University, Hyderabad, India, for providing a part of their RVW database, and to the authorities of the National Institute of Mental Health and Neuro Sciences, Bangalore, India, for providing facilities for recording APP and RVW signals. The authors also express their appreciation to the reviewers for constructive suggestions on the improvement of the manuscript.

\section{REFERENCES}

[1] M. F. O'Ranke, "The arterial pressure pulse in health and disease," Amer. Heart J., vol. 82, pp. 687-702, 1971.

[2] J. R. Cox, F. M. Nolle, and R. M. Arthur, "Digital analysis of electroencephalogram, the blood pressure wave and the electrocardiogram," Proc. IEEE, vol. 60, pp. 1137-1164, Oct. 1972.

[3] T. E. Lee and L. Y. Wei, "Spectrum analysis of human pulse," IEEE Trans. Biomed. Eng., vol. BME-30, pp. 348-352, 1983.

[4] R. L. Martino and W. L. Risso, Jr., "An arterial blood pressure preprocessor using a combined analog and digital signal processing method," in Proc. 7th New England (Northeast) Bioeng. Conf., Mar. 1979, pp. 267-27.

[5] J. Y. Lee and J. C. Lin, "A microprocessor based noninvasive arterial pulse wave analyzer," IEEE Trans. Biomed. Eng., vol. BME-32, pp. 351-355, June 1985.

[6] G. Stockman, L. Kanal, and M. C. Kyle, "Structural pattern recognition of carotid pulse waves using a general waveform parsing system," Commun.ACM, vol. 19, p. 608, 1976. 
[7] H. R. Warner, "Computer techniques for study of circulation," Comput. Biomed. Res., vol. II, ch. 10, pp. 249-251.

[8] T. A. Pryor, "Some techniques for extraction of useful features from biological signals," in Proc. 5th Hawaii Int. Conf. Syst. Sci., Comput. in Biomed., Honolulu, HI, 1972, p. 1.

[9] M. E. DeBakey and D. H. Glaeser, "Waveform analysis of the central arterial pressure using a technique of data compression," J.Assoc. Adv. Med. Instrum., vol. 6, p. 60, Jan. 1972.

[10] G. Avanzolini and P. Barbini, "Comparative evaluation of three online identification methods for a respiratory mechanical model," IEEE Trans. Biomed. Eng., vol. BME-32, pp. 957-963, Nov. 1985.

[11] A. Mukherjee et al., "Computer analysis of breathing patterns in acutely ill patients," in Proc. 8th Northeast Bioeng. Conf. 1980, pp. 403-406.

[12] S. Abbound, I. Bruderman, and D. Sadeh, "Frequency and time domain analysis of airflow breath patterns in patients with chronic obstructive airway disease," Comput.Biomed. Res., vol. 19, pp. 266-273, 1986.

[13] M. V. Rao, D. C. Reddy et al., "The inspiration and expiration DFT spectra for the detection of airwavs obstruction in human beings." Signal Processing, vol. 13, pp. 255-265, Oct. 1987.

[14] D. F. Elliot, Handbook of Digital Signal Processing, Engineering Applications. New York: Academic, 1987.

[15] H. Akaike, "Canonical correlation analysis of time series and use of an information criterion," in System Identification: Advances and Case Studies, X. Mehra and X. Lanoaints, Ed. New York: Academic, 1979, pp. 27-987.

[16] J. L. Shanks, "Recursive filters for digital processing," Geophys., vol. 32, pp. 32-35, Feb. 1967

[17] K. Steiglitz and L. E. McBride, "A technique for the identification of linear systems," IEEE Trans.Automat. Contr., vol. AC-10, pp. 461-464, Oct. 1965.

[18] K. Fukunaga, Introduction to Statistical Pattern Recognition. New York: Academic, 1972.

[19] T. Godfried and X. Toussaint, "Bibliography on estimation of misclas- sification,” IEEE Trans. Inform. Theory, vol. IT-20, July 1974 (Part II)

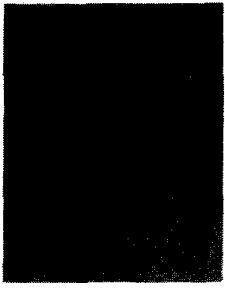

I.S.N. Murthy received the Ph.D. degree from the Indian Institute of Science, Bangalore, in 1968.

He was a Professor in the Department of Electrical Engineering at the Indian Institute of Science. He published papers on control theory, pattern recognition, and ECG processing. His interests included biomedical signal processing, biorobotics, and biocybernetics.

Dr. Murthy was the Editor of the Medical and Life Sciences Engineering Journal of the BME Society of India. He passed away in March, 1992.

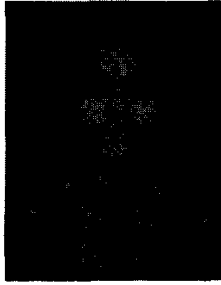

$\boldsymbol{G}$. Sita received the B.Tech. degree in electronics rom Madras Institute of Technology, Madras, an the M.Sc. (Eng.) degree from the Indian Institute of Science, Bangalore, in 1992.

From 1983 to 1990 she was a Scientific Assistant in the Department of Electrical Engineering, Indian Institute of Science, and currently is a Technical Officer in the same Department. Her research interests are in the areas of biological signal processing, medical instrumentation, and application of artificial neural networks. 